\title{
Giacomo Casanova et Cecille von Roggendorff
}

\author{
lettres de sa dernière correspondante
}

\author{
Elena Grazioli
}

\begin{abstract}
Ce texte cherche à analyser l'échange épistolaire entre Giacomo Casanova et Cecille von Roggendorff, à partir du 6 février 1797 jusqu'à la mort de Casanova, le 4 juin 1798. Cette correspondance encadre non seulement un rapport précepteur-écolière, mais, peu à peu, le vieux libertin agit comme un père inquiet pour sa fille. Nous essayerons de suivre les étapes de cette connaissance réciproque à travers les vicissitudes quotidiennes, les maladies de Casanova et les états mélancoliques de Cecille, la tentative de ce dernier de la placer comme dame d'honneur chez les filles $d u$ duc Pierre de Curlande. Le compte rendu lettre par lettre met l'accent sur les interlocuteurs et permet une mise à jour de la narration que l'auteur de l'Histoire de ma vie a consciemment décidé d'arrêter avant ses dernières années passées à Dux.
\end{abstract}

This essay attempts to analyze the exchange between Giacomo Casanova and Cecilia von Roggendorff, during the two years before Casanova's death in Dux, Bohemia (4 June 1798). Such correspondence represents the step-by-step evolution of a teacher-pupil relationship into a father-daughter one, with the old libertine getting increasingly worried about her. Our analysis follows such mutual exchange through everyday matters such as Casanova's illness and Cecilia's melancholy, the former's attempt to place her into the court as the duke Pierre de Curlande's maid of honor. By means of a letter-by-letter account, some light is shed on the interlocutors and Casanova's narration in his Histoire de ma vie, which omits his last years spent in Dux.

Voilà donc le diable, devenu ermite, parle un langage auquel nul ne peut trouver à redire. Suivons-le dans sa courte carrière de directeur spirituel et intellectuel.

—Joseph Pollio et Raul Vèze, Pages Casanoviennes 
La première lettre écrite par Cecille von Roggendorff (1775-1814) à Giacomo Casanova (1725-1798) remonte au 6 février 1797, avec celle-ci commence un échange épistolaire qui se limite à la dernière année de vie du libertin. ${ }^{1}$ Cecille fut — sans oublier Élise von der Recke - sa dernière correspondante: Casanova meurt le 4 juin 1798 sans jamais avoir eu la chance de la rencontrer. ${ }^{2}$ Cependant, le compte rendu lettre par lettre met l'accent sur les interlocuteurs et permet une mise à jour de la narration que l'auteur de l'Histoire de ma vie a consciemment décidé d'arrêter avant ses dernières années passées à Dux. ${ }^{3}$ Ainsi, d'une certaine manière, le récit est soutiré à Casanova qui n'est plus le chef, ou il ne l'est complètement, de la structure narrative, il n'interagit pas avec celle-ci en mettant en relief ou en cachant certains faits, en ôtant ou en ajoutant ce qu'il désire: l'horizon épistolaire conditionne et enlève le masque du narrateur, le vieux Casanova est toujours mis en scène malgré lui. D'ailleurs, le type humain n'exclut point une autre version de l'histoire, une narration différente.

Le récit épistolaire et le récit autobiographique se reposent sur différentes règles de genre; grâce aux réflexions de Lejeune, dans Le pacte autobiographique (1975), nous apprenons que le récit autobiographique exhorte une intention de sincérité, mais il n'oblige pas à l'exactitude, comme les conditions du pacte référentiel illustré par le professeur le soulignent. La correspondance par lettre se structure sur l'émotion du moment et sur un dévoilement honnête de notre être, tandis que l'élaboration du vécu appar-

1. Caecilie von Roggendorff (Vienne, 28 octobre 1775-Vienne, 27 novembre 1814), fille aînée légitime née en secondes noces de son père Ernst von Roggendorff avec Wilhelmine Friderici de Friedwald. J'ai choisi de garder la graphie "Cecille" avec laquelle elle-même signe la correspondance.

2. On peut lire les lettres d'Élise von der Recke (1754-1833) en français dans le volume Ravà 1912, ou en italien dans le livre Orsenigo 1997. Pour approfondir le rapport entre Giacomo Casanova et Élise von der Recke, on peut lire LeEflang 2000, 133-43 ou ma version italienne: Elena Grazioli, Umori e lettere inglesi delle confidenti di Giacomo Casanova, dans Kornevva 2019, 137-50.

3. Aujourd'hui nous ne disposons pas d'une étude critique qui analyse, dans sa structure, les années que Giacomo Casanova a passées à Dux; de matière romanesque le volume VAssalli 2002 et Bartolini 1994, même si ce dernier se présente comme plus global, parce qu'il inclut aussi les années de son retour à Venise et celles passées à Vienne; la contribution d'Helmut Watzlawick, Les tristesses de Dux: critique d'un mythe, dans Pizzamiglio 2001, 67-77 cherche à renverser, ou tant au moins à atténuer, les aspects négatifs de la sénilité de Casanova; enfin, essentiel pour l'encadrement historique, l'étude Cengi a rott i 1990. 
tient à d'autres horizons narratifs, ainsi l'explique Bruno Capaci dans le livre Giacomo carissimo . . . (2019): "La mediazione della vita portava Casanova a inseguire l'hasard e soprattutto a esibire quella teatralità che fa di lui un attore e un affabulatore nel medesimo tempo. Se ci pensiamo bene questo è il processo che conduce Casanova prima al récit da salotto, poi allistant book e infine all'autobiografia vera e propria". Également, nous ne devons pas oublier certaines caractéristiques fondamentales de la correspondance qui se manifestent à la fin du siècle: si les missives connaissent une grande fortune dans les pratiques d'écriture au XVIIIème siècle (à voir, par exemple, l'essor des romans par lettres) et elle jouent un rôle stratégique comme dispositif narratif et dramaturgique, il faut préciser aussi que le modèle qui s'impose progressivement dans la correspondance au siècle des Lumières est celui de la lettre anglaise; ${ }^{5}$ éloignée des éléments piquants et provocateurs, la lettre anglaise s'encadre bien dans la définition de correspondance familiale qui répond à la fonction de maintenir les relations entre personnes liées d'un rapport de confiance et d'une caractère commun des sentiments avant que d'intérêts; ${ }^{6}$ une forme du discours plus immédiate, mais non incontrôlée, qui nous présente la rhétorique du quotidien déclinée dans des caractères de spontanéité d'un réalisme narratif privé d'euphémismes et d'ornements, qui assez fréquemment débouche sur la confiance galénique. $^{?}$

4. Bruno Capaci, Una sirena con la chitarra e con la penna, dans CAPACI-GraZioli 2019, 36-60, 53.

5. La référence est aux lettres de Voltaire, cf. Voltaire 1830.

6. Je fais référence au modèle de Vincent Voiture (1598-1648), qui représente encore très bien l'esprit précieux du XVIIème siècle dans la diversité de ses missives. Malgré cela, les (Euvres de M. de Voiture (cf. Voiture 1650), composées par trois-quarts de lettres privées et familières, connaissent un immense succès: elles sont reconnues par le public et aussi par les lettrés. La publication provoque un débat autour de la littérarité du discours épistolaire et de la modalité avec laquelle ce canon s'est formé au cours du XVIIème siècle. Pour approfondir on peut prendre en examen les contributions de Giorgio Ronconi, Aspetti della lettera familiare nel Settecento. La corrispondenza tra Egidio e Marco Forcellini e la prima edizione dell'epistolario zeniano, dans Chemello 1998, 229-43; Rollin 2009, 251-63; Grosse 2017; Forner 2020.

7. Cf. Bruno Capaci, Modelli e occasioni epistolari del Settecento, dans Viola 2011, 73-89, 79: "Le notizie sulla salute e sulla quotidianità sono d'obbligo, ma è altrettanto vero che farne argomento non marginale di conversazione, non temere affatto di parlarne per particolari, ma anzi indulgere al privato in alternativa a scambi di riflessioni politiche, letterarie o filosofiche, è cosa del tutto nuova e peculiare del secondo Settecento". Dans la même contribution (Viola 2011, 
Fille d'Ernst von Roggendorff et de Wilhelmine Friderici, ${ }^{8}$ Cecille von Roggendorff est une jeune comtesse à l'histoire malheureuse: orpheline de mère et de père (sa mère meurt le 31 octobre 1785 et son père le 3 septembre 1790), elle passa son enfance dans un couvent de Salésiennes; elle réussit à entrer, à l'âge des 17 ans, dans l'ordre des Saint-Augustin comme chanoinesse de Hale, ${ }^{9}$ mais cela ne l'empêcha pas de passer une jeunesse dans la détresse. Vers lâge de vingt an sa vie sembla lui montrer un visage moins funeste, grâce à son engagement avec le baron de Vecesey. Malheureusement, le sous-lieutenant autrichien mourut dans la bataille de Bassano, le 17 novembre 1796. Seule et sans affection, Cecille trouva un refuge chez sa cousine, la comtesse Török, ${ }^{10}$ à Kaschau (Cassovie). Trois mois après la perte de son fiancé, elle écrivit à Casanova pour lui demander amitié et protection.

Dès les premières lignes, en s'excusant par avance pour son hardiesse, Cecille offre à Casanova un "commerce de lettres suivie" qui se base sur l'argument de transitivité:11 puisque vous étiez l'ami de mon père (Cecille l'a appris grâce à son frère Ernst ${ }^{12}$ ) accordez-moi aussi ce titre; ${ }^{13}$ et, en adop-

41-72), l'étude de Fabio Forner, Per una storia dell'epistolografia nel Settecento (avec une référence précise aux observations au sujet des raisons d'un renforcement des communications dans le panorama européen), me semble fondamental.

8. Le comte Ernst von Roggendorff (1714-1790), épousa, très jeune, Anna Zaruba qu'il abbandona peu de temps après le mariage pour vivre avec sa maitresse, Wilhelmine Friderici (-1785); avec elle il aura deux enfants, Ernst et Ernestine. À l'âge de soixante ans, en 1774, à la mort de son épouse légitime, il épousa Wilhelmine; en secondes noces naquirent Caecilie et cinq autres enfants.

9. Titre conféré de l'impératrice Marie-Luise, épouse de Léopold II, le 4 mai 1792.

10. La comtesse Aloisia Török née Roggendorff en 1754, épouse de Louis Török.

11. "La transitivité est une propriété formelle de certaines relations qui permet de passer de l'affirmation que la même relation existe entre les termes $a$ et $b$, et entre les termes $b$ et $c$, à la conclusion qu'elle existe entre les termes $a$ et $c$ : les relations d'égalité, de supériorité, d'inclusion, d'ascendance, sont des relations transitives" ainsi Perelman-Olbrechts-Tyteca 2008, 305.

12. Le comte Joseph Albert Ernst von Roggendorff (6 novembre 1772-1777 avril 1842), officier autrichien et frère de Cecille. Pendant la campagne militaire du 1793, il se distingua dans la prise de Marchiennes (30 octobre). Il passa une période de sa vie à Dux, en fonction d'administrateur adjoint du château et des écuries; c'est là qu'il rencontra Casanova. Il reçut une pension grâce à son titre d'Oberstleutnant et il épousa la comtesse Petrovitch avec qui il aura cinq enfants.

13. Casanova évoque brièvement l'image du père de la Roggendorff dans l'Histoire de ma vie, et dans son Précis de ma vie: "[. . .] et Vienne où j’ai connu le comte 
tant un raisonnent a fortiori, ${ }^{14}$ elle justifie l'élan de l'écriture à plus forte raison' puisqu'il provient des élans d'un cœur malheureux. Avant de poursuivre, il est nécessaire de préciser que Casanova ne s'accoutuma jamais à la langue allemande, même s'il a passé vingt-ans en Bohême, à Dux; Cecille, née à Vienne et encore très jeune, ne pouvait connaître l'italien bien que, par admiration envers Casanova, elle se déclara disposée à l'apprendre ${ }^{15}$ - l'échange épistolaire se déroula donc sur un terrain commun: les lettres ont été écrites en français. Nous avons trente-trois lettres de la Roggendorff adressées à Casanova et deux réponses de ce dernier, parmi lesquelles le Précis de ma vie; ces missives sont conservées à l'Archive d'État de Prague (SOA Praha). J'ai suivi, en les citant, le catalogage introduit par Bernard Marr, le même utilisé dans une brochure qui circule uniquement entre casanovistes et qui contient la transcription diplomatique des lettres manuscrites (Cf. Lettres de Cécile de Roggendorff à G. Casanova, texte établi et annoté par Marco Leeflang et Monique Grenier, Utrecht-Paris, s.n., 1996). Il faut préciser que la publication de cet échange épistolaire remonte à l'an 1926, en version complète et en langue française, dans le Vlème volume des Pages casanoviennes, intitulé La dernière amie de Jacques Casanova: Cécile de Roggendorff (1797-1798), par Joseph Pollio e Raul Vèze, et nous le retrouvons aussi dans une récente édition de poche, Lettres d'amour à Casanova, par l'historien Alain Buisine; mais les deux éditions présentent une normalisation totale du texte. ${ }^{16}$ Le missives de Cecille ont été aussi en partie traduites et publiées par Aldo Ravà et Gustav Gugigtz dans la collection Frauenbriefe an Casanova (1912), mais elles n'ont jamais été traduites en italien. Dans ce travail, j'utilise ma transcription à partir des autographes: je suis intervenue uniquement dans les cas où l'orthographe incorrecte créait des problèmes de compréhension du lemme et de la signi-

Roggendorf" (cf. Casanova 2018/3, 1197).

14. En ce qui concerne le raisonnement a fortiori, je fais à nouveau référence à Perelman-Olbrechts-Tyteca 2008.

15. "Si tot que je verai l'abbé d'Este, je lui ferai vos tendres compliments, mais pour la langue italienne je ne sais comment faire pour l'aprendre, d'après que mes entrevûes avec l'abbé d'Este sont tres rares puisque je vis toujours à la campagne ou [où] je ne voit j'amais du monde. Je lui ai écrie [écrit] pour une grandmere [grammaire] italienne, la reponse fuit [fut] qu'il n'en avoit point" (Marr 8-26).

16. "Nous ne respecterons pas l'orthographe de Cécile de Roggendorff qui la respectait, elle, médiocrement. Prévenue sans doute que Casanova ne connaissait pas l'allemand, ne connaissant pas elle-même l'italien, elle dut s'obliger à écrire en français. Comme tous les correspondants de langue germanique de Casanova, elle y réussissait assez mal": PolLio-VÈze 1926, 4. 
fication de la phrase; les interventions de corrections ont été faites dans la marge, entre crochets, pour permettre de comprendre à travers ces passages les compétences lexicales, orthographiques et syntaxiques d'une jeune fille semi-savante dont le français est contaminé par l'allemand et emprunte à l'oralité. ${ }^{17}$

Des attestations d'estime de matrice épidictique se décèlent dès le début: 18 "Vos merittes, votre age, votre experience m’enspire la plus grande veneraison [vénération]", ${ }^{19}$ de même, apparaît immédiatement le chagrin de ne pouvoir pas jouir de la compagnie du Chevalier de Seingalt (plus chanceux son frère Ernst qui se trouve à Dux ${ }^{20}$ dont le manque est pallié à travers l'écriture. Cecille n'oublie pas de s'excuser pour son "mauvais stil que ecriture"; d'ailleurs, comme nous l'avons anticipé, elle nait à Vienne en 1775 et elle est donc de langue maternelle allemande. La captatio benevolentiae envers Casanova se termine encore par un argument de transitivité qui a un fondement rhétorique assez fallacieux: Cecille construit, dans un premier moment, l'éloge du comte de Waldstein, qu'elle croit être le plus aimable entre les hommes, et, précisément au nom de cette amabilité du comte, Casanova devrait se montrer obligeant et accepter cet échange épistolaire. Comment donc s'y soustraire? D'autant plus que les dernières lignes

17. À propos de l'écriture féminine indispensable le livre de Plebani 2019. Sur la langue française du XVIIIème siècle on peut lire BRUnOT 1905, en particulier la deuxième partie du huitième volume, rédigé par Alexis François. En ce qui concerne plus précisément l'orthographe de la langue française cf. Dictionnaire historique de l'orthographe française (Paris, Larousse, 1995), avec des références ponctuelles à CATACH 2001.

18. Au genre épidictique est dédié le neuvième chapitre de Réthorique II d'Aristote, la finalité est celle de "riuscire a migliorare (o peggiorare) la realtà di cui si parla e ciò deve essere realizzato all'interno del discorso, il che significa, in ultima analisi, attraverso mezzi linguistici" ainsi Piazza 2015, 85. Dans le domaine de la rhétorique, on peut prendre en examen ces contributions: BAtTistini-RAImondi 1990; Mortara Garavelli 2018; Piazza 2004.

19. Lettre de Cecille von Roggendorff à Giacomo Casanova de Cassovie, le 6 février 1797 (Marr 8-25).

20. Les rapports entre Ernst et Casanova étaient houleux, comme le démontre une lettre de Casanova écrite de Dux, le 2 juin 1797: "Ditemi perché non imitate il Conte vostro padre che si faceva rispettare da tutti. Ditemi perché tutti dicono male di voi. Ditemi perché tutti dicono che siete un bevitore, un attaccabrighe, uno che fa dei debiti che non può pagare [. . .]" ainsi Casanova 1969, 424-27, 425. 
de la missive esquissent un premier portrait de Mlle Roggendorff, aussi belle qu'Ernst, bien que plus maladroite. ${ }^{21}$

J'ai déjà précisé que les réponses de Casanova ne sont qu'au nombre de deux (y compris le Précis de ma vie), toutefois, grâce aux informations que la comtesse nous délivre en reprenant certaines phrases du Chevalier, nous pouvons reconstruire, en partie, la tonalité de la correspondance qui se base sur une affection sans la moindre équivoque malgré ses tendres élans. ${ }^{22}$ À mon avis, il n'est pas possible de construire un parallèle avec certaines déclarations de Manon Balletti envers Casanova, qui sont ellesaussi très tendres, mais qui présentent des acceptions bien plus allusives et rassemblent plutôt à ce qu'une épouse est disponible à accorder. ${ }^{23}$

Si nous respectons le statut de vérité du genre épistolaire, la lettre de Cecille a ému Casanova jusqu'aux larmes; et nous trouvons de la douceur même chez le vieux libertin, précisément dans le ton avec lequel il dispense ses conseils à sa jeune écolière. Casanova crée le rôle de précepteur parce qu'il exige encore une position de suprématie, il veut encore être le protagoniste, même s'il est rappelé sur scène par une femme et même si à Dux ne reste des salons qu'une petite table. Le libertin devient précepteur à un certain âge, exactement comme pour la protagoniste des Lettres de Ninon de Lenclos au marquis de Sévigné (1750), ${ }^{24}$ mais Casanova n'est pas si audacieux et au même temps il ne se transforme guère en percepteur sur le modèle de Giuseppe Parini; ses réflexions philosophiques sont tirées et

21. Nous pouvons lire le portrait plus approfondi que Cecille fait d'elle-même dans Marr 8-110.

22. Nombreuses, dans la correspondance, les occurrences de "Vous dites que" auxquelles fait même suite, dans certains cas, la citation directe du discours de Casanova: "Vous vous nommé depuis l'âge décrepit un exorther" (mien l'italique).

23. Je fais référence ici à une lettre de Manon Balletti à Giacomo Casanova, du 10 septembre 1757, dans laquelle elle affirme: "[. . .] sognate tutto quello che vi piacerà e un giorno forse, se la fortuna lo vorrà, confermerò la vostra immaginazione" (cf. CAPACi-Grazioli 2019, 162). En ce qui concerne la figure de Manon Balletti et son rapport avec Casanova, qui déboucha sur des documents de mariage, la référence renvoie toujours à l'essai de Bruno Capaci, Una sirena con la chitarra e con la penna, dans CA PACI-Grazioli 2019, 36-60.

24. À voir en propos la contribution de Bruno Capaci, 'Lettres de Mademoiselle Ninon de Lenclos au Marquis de Sévigné. Una maschera epistolare della femme au dixhuitième siècle, dans For ner 2017, 223-37. 
de la théorie des Lumières et du discours concernant le plaisir. ${ }^{25}$ Encore une fois, il se raconte face à un public, en apprenant même à dévoiler les contenus qui n'amusent pas. Il a fait suivre à l'éloge de la vertu des conseils qui concernent la rectitude des principes, mais il les a exposés de manière si charmante à susciter le désir de s'y conformer; ainsi Joseph Pollio e Raul Vèze dans La dernière amie de Jacques Casanova: "Pourquoi ne pas constater simplement — sans la moindre teinte d'admiration — la dualité de cet être, de tout être humain, conscient de la grandeur de son esprit, de la faiblesse de son corps et des ses sens? Ainsi qu'il l'écrivit peu de temps après à Ernest, il a vu une tâche sévère à remplir, il n'a pas cru pouvoir s’y dérober" (PoLLIO-VÈzE 1926, 11). Toutefois, Cecille - nous pourrons dire en tant que jeune $^{26}$ - oppose une lex potentior selon laquelle la nature "est celle du sentiment du cœur dont l'homme n'est jamais qu'à demie le maitre”; 27 elle se débarrasse donc bientôt du joug de la raison. Comme une écolière diligente, la Roggendorff veut mériter cet échange des lettres, mais, grâce à sa franchise, ce qu'elle ressent s'oppose aux maximes casanoviennes et elle ne dissimule point sa réprobation: "je connoit la vertu et des gens vertueux qui cependant ne sont point heureux". ${ }^{28}$ Par contre, elle est plus disponible à se consacrer aux études: Casanova doit lui avoir demandé d'apprendre l'italien (ou simplement conseillé) par l'intermédiation de l'abbé d'Este; ${ }^{29}$ Cecille

25. "Le percezioni dei sensi sono la garanzia stessa dell'esistenza, la condizione sine qua non il piacere vi sarebbe assente. D'altra parte, questa concezione, debitrice del Traité des sensations (1754) di Étienne Bonnot de Condillac (1714-1780) e della filosofia che gli è propria in senso più ampio, si allontana dall'idealismo per avvicinarsi a un'origine materialistica della conoscenza del proprio io. Si apprende rielaborando le sinestesie dell'esistenza con il fine di ri-conoscere e ri-conoscersi" ainsi Elena Grazioli, Umori e lettere inglesi delle confidenti di Giacomo Casanova, dans Korneeva 2019, 139.

26. Dans son analyse des ethe (Rhétorique II, 12-17), Aristote avait distingué trois différents 'tipi di carattere' sur la base de l'âge: jeunes, vieux et hommes mûrs; “i caratteri dei giovani sono schematicamente rappresentati come opposti [. . .]. Se i giovani sono tendenzialmente più coraggiosi, impulsivi, passionali e collerici, i vecchi, invece, sono più inclini ad avere paura e sembrano più temperanti, perché hanno desideri più deboli o si sono abituati a tenerli a bada" ainsi Prazza 2015, 108.

27. Lettre de Cecille von Roggendorff à Giacomo Casanova, le 10 mars 1797 (Marr $8-26)$.

28. À propos de la connexion entre vertu et bonheur, on peut faire référence aux observations de Michel Delon, dans les chapitres Bonheur et Bonheur, suite, dans Delon 2011, 245-55.

29. L'abbé italien Vittorio d'Este était professeur à l'académie de Kaschau. 
s'excuse immédiatement de ses rares visites au religieux et pour n'être pas parvenue à se procurer une grammaire de la langue italienne, ainsi elle ne peut démontrer son empressement à suivre les conseils de son précepteur. ${ }^{30}$ Mais pour remercier son pédagogue, en répondant aux vers qu'il doit lui avoir envoyé, Cecille formule sa gratitude en alexandrins. ${ }^{31}$

La seule réponse de Casanova qui nous est parvenue, en excluant bien évidemment le Précis de ma vie, c'est une lettre du 22 mai 1797.32 Cette fille lui a fait l'honneur de démontrer son estime et sa confiance gratuitement parce qu'elle n'aurait pu savoir s'il les mériterait (elle ne le connaissait pas en personne). Casanova, de son côté, lui montre sa reconnaissance à travers certains préceptes qu'elle peut prendre comme des conseils: "A l'age de vingt ans vous devez commencer à dédaigner tous les pardons imaginables, vous ne devez pas en exiger; vous ne devez jamais en avoir besoin. [. . . ]. Faitesvous respecter, et sachez noblement mépriser ceux qui vous manquent, mais sans leur faire voir votre mépris, [. . .]. Bornez vos désirs et banissez de vos rêveries tous les châteaux en Espagne" (Marr 40-3). La tonalité de précepteur qu'assume Casanova est la même que nous retrouvons dans la lettre à Maria Gioseffa, qui remonte à des années bien antérieures. ${ }^{33}$ Sa passion d'apprendre est devenue celle d'enseigner; il s'agit, cependant, d'un élan de nature particulière car, en tenant compte de ce que le prince de Ligne affirme assez méchamment, notre Chevalier se sentirait excité à cette tâche uniquement par l'air ravissant des jeunes filles (Ligne 2003). Toutefois, je crois que dans les attentions et les soins de Casanova envers Cecille, il faudrait plutôt parler de l'affection d'un père. D'ailleurs, Cecille elle-même ne configure leur rapport que dans ces termes: "Vous m'aimés comme un pere, dites vous? Qu'elle est donc la tendresse que je Vous porte, est elle moins

30. "Si tot que je verai l'abbé d'Este, je lui ferai vos tendres compliments, mais pour la langue italienne je ne sais comment faire pour l'aprendre, d'après que mes entrevûes avec l'abbé d'Este sont tres rares puisque je vis toujours à la campagne ou [où] je ne voit jamais du monde. Je lui ai écrie [écrit] pour une grandmere [grammaire] italienne, la reponse fuit [fut] qu'il n'en avoit point" ainsi Cecille von Roggendorff à Giacomo Casanova, le 10 mars 1797 (Marr 8-26).

31. "Le plaisir cher ami que tu m'as procuré // me fera bénir cet heur fortuné // ou le ciel te fît naitre, vrai predestiné // qui comble de bonheur des coeurs affligé" (Marr 8-26).

32. Lettre de Giacomo Casanova à Cecille von Roggendorff de Dux, le 12 mai 1797 (Marr 40-3).

33. Lettre de Giacomo Casanova à Maria Gioseffa, petite comtesse Lamberg, de Dux, le 25 février 1791 dans Casanova 1969, 312-15. 
vif?"34 Casanova semble se définir dans un noyau familial et il intervient à l'égard de Cecille exactement à cause des préoccupations qu'elle nourrit pour son frère, il me paraît même qu'il veut presque se substituer à Ernst, en condensant sur lui-même la famille que Cecille n'a plus.

Casanova n'a seulement accordé, au début, sa disponibilité à entretenir une correspondance avec Cecille, mais, avec un égal empressement, il s'est mobilisé pour lui faire obtenir une place à la Cour, grâce à l'intercession de la princesse Clary, la fille de son ami le prince de Ligne, ${ }^{35}$ afin de lui garantir un apanage suffisant pour lui assurer une certaine indépendance. Puisque Cecille répond immédiatement que sa tante Salm n'est pas d'accord, ${ }^{36}$ Casanova cherche à la placer comme dame d'honneur chez les filles du duc Pierre de Curlande. ${ }^{37}$ Le tuteur de Cecile trouve erronée cette decision et, au cas où Cecille se résout à continuer dans cette voie, il la prie de s'assurer, avant d'abandonner son bénéfice ecclésiastique, que ce duc lui fournira une petite pension, même si elle ne sera pas adaptée à la mansion prévue, pour qu'elle ne reste pas sans rémunération. Cecille, si jeune qu'elle soit, ne peut se rendre compte qu'une telle proposition fait offense au duc de Curlande, mais Casanova garantit pour elle la pureté de son cœur et de son esprit. ${ }^{38}$ La totalité de l'échange épistolaire entre la Roggendorff et Casanova suit la trace des obstacles et des résolutions en ce qui concerne le départ de Cecille; entre autres, ce démarrage lui permettrait une visite

34. Lettre de Cecille von Roggendorff à Giacomo Casanova de Kazmer, le 22 octobre 1797 (Marr 8-33).

35. Marie-Christine-Claudine-Léopoldine-Philippine de Clary, née de Ligne (17571830), épousa le 31 mai 1775 le comte Jean Népomucène Clary. Elle était amie de l'empereur et brilla à la cour autrichienne et pour son esprit et pour son talent de chanteuse d'opéra. Elle fut longtemps correspondante de Casanova (plusieurs lettres ont été retrouvées à Dux) et elle lui presenta son père, le prince de Ligne, à Toeplitzl, pendant le mois d'août de l'année 1790.

36. Raphaele von Salm-Reifferscheid-Krautheim (1726-1807), comtesse Roggendorff, sœur du père de Cecille - elle prendra le patronyme de son époux une fois mariée - elle est la mère du cardinal Franz Xaver Salm.

37. Duc Pierre de Courlande (1724-1800), fils d'Ernest de Curlande. Le duc avait dû renoncer à son duché en faveur de Catherine II qui lui laissa une rémunération de cinquante mille ducats. Il vivait avec sa troisième épouse, Dorothea von Medem (épousée en 1779), sœur ainée d'Élise von der Recke, et ses quatre filles: Wilhelmine, Paolina, Johanna, et Dorothea.

38. À ce propos, voir la lettre que Casanova écrivit au duc Pierre de Curlande de Dux, le 20 janvier 1798, dans Casanova 1969, 442-44. 
à Casanova à Dux. Seulement peu après la mort du Chevalier, Cecille obtiendra définitivement l'emploi et quittera Hale.

Le chevalier de Seingalt ne s'était pas donc trompé quand il avait promis à Cecille, à travers une réminiscence de cabaliste - il l'avait 'lu' dans les livres destinés -, qu'elle aurait été heureuse. Cecille lui répondit avec une antanaclase: "je crois pouvoir dire [de] sentire le commencement de cette felicité quand je reçoit de Vos lettres" ${ }^{39}$ Toutefois, dès la fin du mois d'avril 1797, dans l'écriture de Cecille une profonde mélancolie s'était manifestée:

Hélas, lorsqu' aucupés [occupée] d'un serieux retour sur moi même, sur ma situation presente et future, j'inore [j'ignore] si j'orai [j'aurai] assés de fermeté pour me soumaitre [soumettre] à tout le poix [poids] des meaux [maux] qui va peut être me couvrire. Mais étant convaincue que la pactience est la seule chose qui existe parmis les hûmeuns [humains] qui merite le titre de vertu veritable, toutes autres n'étant qu'effait [effet] du temperament, je crois que, gémissant depuis l'age de 9 ans dans les larmes, je sorai souffrir encore les derniers épreuves avec resignation.

(Marr 8-28)

Si d'un côté Casanova regrette sa jeunesse et sa vigueur physique, la Roggendorff, dans la fleur de l'âge, ne semble pas être touchée par la négritude, au contraire, elle l'invoque comme qui, en proie à la mélancolie, à l'humeur de la bile noire, souffre davantage pour le désespoir intense et la dépression que pour les effets de la déchéance physique. Le soleil noir affecte négativement le corps, dont les symptômes sont toutefois de plus facile définition que les souffrances de l'âme:

La pâleur de mon visage et mon humeur un peu triste, melancolique, inquiète mes amis, l'on m'ordonne de me rendre au Bain de Rancke pour prendre des fortificasions [fortifiants]. Mon depart est fixée au 30 de ce mois. Je Vous avoûe me preter avec asses [assez] mouvaise grace à la volonté des medecins puisque le delabrement de ma santé na rien qui m'inquiète, ô non, il me charme. ${ }^{40}$

39. Lettre de Cecille von Roggendorff à Giacomo Casanova du 30 avril 1797 (Marr $8-28)$.

40. Lettre de Cecille von Roggendorff à Giacomo Casanova de Cassovie, le 25 juin 1797 (Marr 8-113). 
Je me suis plainte dans ma dernier d'un gros hrume [rhume], mais je ne me rappelle pas avoir parlé de santé delabré. Comme je Vous ai écrit que je me preparoit pour aller aux bains de Ranck, alors j'etoit malade sens [sans] qu'on peut [pût] donner un nom à ma maladie, l'ame ne se devine. Vous ne soriés croire comme le chagrien influe sur le phisique: quelques jours de souffrance, et il faut des semaines pour me remettre [ . . ].1

Les descriptions des bains thermaux rappellent à la mémoire certains tableaux esquissés par la poétesse vénitienne, assidue des salons illuminés, Caterina Dolfin (1736-1793), destinés au patricien Andrea Tron (17121785), son futur époux, qui remontent à quelques années plus tôt. ${ }^{42}$ Cependant, Cecille ne s'attarde pas sur les aspects mondains des bains et elle ne rappelle pas la présence de la société des médecins, dont on a des descriptions plus amples dans les contes de la Dolfin. Nous ne devons pas oublier que Philippe Pinel (1745-1826) — médecin précurseur de la psychiatrie, exerçant à cette période à la Salpêtrière - fait l'éloge des avantages des eaux thermales, dans un article concernant la mélancolie, pour les troubles provoqués par cette forme de délire partiel..$^{43}$ Débile, moins réactive, selon les stéréotypes traditionnels, la femme risque de perdre plus facilement le goût de l'existence. La parole 'vapeurs' s'est imposée au XVIIIème siècle pour indiquer précisément le joug de l'ennui sur la vita activa, capable d'arrêter soit des procès psychologiques soit physiques. Joseph Bressy en décrit la symptomatologie dans son traité Recherches sur les vapeurs, qu'il publia précisément à cette époque (1789).

L'écoute du malaise des autres est dominé par l'argument de réciprocité, qui se balance entre un pôle positif, représenté par une avantageuse correspondance qui rappelle Cicéron "Longin se porte mieux et Zénobie n’a plus de peines" ${ }^{44}$ et un pôle négatif selon lequel l'influence mutuelle assume des nuances saturnines, bien évidentes dans la brillante réplique de Voltaire souffrant pour le chagrin de Mme du Deffand: "J'ai le malheur d'être tout

41. Lettere de Cecille von Roggendorff à Giacomo Casanova de Cassovie, le 10 décembre 1797 (Marr 8-38).

42. Pour ce qui concerne la correspondance de Caterina Dolfin, cf. Bruno Capaci, Modelli e occasioni epistolari del Settecento, dans Viola 2011, 73-89, 83 et suiv.; Capaci 1996, 191-228; Damerini 1929.

43. Pour approfondir les bains thermaux, on peut faire référence au chapitre de Jean Starobinski, L'établissement thermal, dans Sta robinski 2012, 114-17.

44. Noms de plume de Casanova, dans le rôle de ministre et précepteur, et de la Roggendorff, reine de Palmyre. 
le contraire de Cicéron: si vous vous portez mal, j'en suis fâché". ${ }^{45}$ Dans les mots de Cecille, cette influence mutuelle est même dépassée: la seule figuration dans la pensée de la mort de son ami est cruelle au point de susciter le désir de négocier des années de sa vie au prix du bien-être de la personne aimée:

Pauvre et resigné ami, que vos lettres me touche, hélas! Je donnerai volontié dix années de ma vie pour racheter à ce prix votre santé; n'alés pas Vous imaginer que ce soit un compliment ou une belle phrace [phrase], c'est le voeu le plus réel, le plus sincère que mon coeur n'ai jamais formée. Vous guérirai, je m'en flatte, j'en suis sûr, et ne me parlés plus de la mort: ce mot me fais un mal affreux, il est cruel de me la faire pleurer si longtems d'avance. Si l'idé[e] que Vous êtes un être mortel ne Vous épouvante pas trop, pensés-y bien toujours, c'est un bon signe à ce que dissent [disent] les bonnes femmes chez les malades, ils aproches [approchent] alors de leur guerrisons [guérison]. ${ }^{46}$

Dans ce cas la 'guérison' de Casanova est plutôt celle des maux de la vie. Les aspects du réalisme dans l'écriture épistolaire se profilent non seulement en cette attention au quotidien, mais aussi dans la présence du présent dans la correspondance. Intéressant du point de vue historique est le jugement que Cecille apporte par rapport à la guerre:

On souhaite la paix, on la dit certaine, mais on semble pourtent [pourtant] prendre des mesures pour continuer la guerre; on commence, d'après les ordres reçues, de preparer toutes choses pour l'insurrection. Le premier de mai tous les comitats doivent s'asambler [s'assembler] et on tiendra une congregation pour prendre des mesures convenables, aussi se trouvent ici tout le monde dans la plus grand consternation: on attent [attend] la post de dimanje [dimanche] avec la plus grand impatience, puisque des lettres de Vienne nous prometes [promettent] des nouvelles sur [sûres]. ${ }^{47}$

45. Lettre de Voltaire à Madame du Deffand du 18 février 1760, dans Voltaire 1832/8 tome 58, 315-17, 315.

46. Lettre de Cecille von Roggendorff à Giacomo Casanova de Vienne, le 17 mars 1798 (Marr 8-15).

47. Lettre de Cecille von Roggendorff à Giacomo Casanova du 20 avril 1797 (Marr $8-27)$. 
La correspondance de Casanova avec Cecille n'est exempte d'une certaine dimension galante: aux égards de Waldstein, Cecille admet être toujours joyeuse en pensant à lui et elle décide de répondre à Casanova qui veut savoir si cette amitié - il doit en avoir perçu le zèle excessif — se fonde sur un "bon raisonnement" ${ }^{48}$ Cecille se confie à sa discrétion en reconnaissant qu'elle n'est pas capable de déchiffrer les sentiments qu'elle nourrit envers le comte, toutefois elle était "enflammé de la plus tendre gratitude" (Marr 8-28). Cecille admet avoir pris des renseignements sur le comte: ses passions, ses goûts, sa figure, ses manières. . Ces mots prononcés par la personne qu'en a fait l'éloge déclenchent en Cecille l'envie de le connaître en personne, bien qu'elle ignore s'il s'agit d'“amitié, reconnoissance, amour ou un autre sentimens qui me parle pour lui" (Marr 8-28). Elle demande à Casanova, probablement pour cette raison, s'il est bien qu'elle désire le voir si ardemment. Cecille, qui ne cesse de s'encadrer dans la figure de l'écolière diligente, prétend qu'on lui montre ses fautes, pour qu'elle puisse se corriger à l'avenir; l'estime envers Casanova l'amènera à suivre ses conseils: "je ne suis ni opiniâtre ni entêté et [je] sais accepter la contraticion [contradiction] quand on l'apuis [appuie] sur des verités esenciele [essentielles]" (Marr 8-28). Cependant, à la fin de la lettre, elle demande exactement à Casanova de rapporter au comte de Waldstein qu'elle est heureuse chaque fois qu'elle pense à lui. Casanova a dû sursauter face au terme amour: le comte de Waldstein a quand même quarante-deux ans, il est célibataire, mais son caractère est peu adapté pour rendre heureuse une jeune fille. Par ailleurs, à ce moment-là, Casanova était outragé par la conduite d'Ernst auquel il avait adressé une lettre âpre contenant des violents reproches. ${ }^{49}$ Il doit avoir réagi ainsi envers Cecille probablement parce qu'il se comporte réellement comme un père craignant les déceptions de sa fille. En effet, la Roggendorff se plaint des tons changés radicalement: "Mais, cruels ami, que m'avés vous dit et qu'elles affreuses verités exposés Vous à mes yeux? Vous déchirés un voile pour me desabuser sur un bonheur imaginaire qui n'ora[aura] jamais lieu, je Vous suis redevable de cette probité, mais sachés que Vous m'avés mal approfondi". ${ }^{50}$ De manière oxymorique Casanova est défini comme un 'ami cruel' puisqu'il l'a détrompée par rapport à des bonheurs qui existaient

48. Lettre de Cecille von Roggendorff à Giacomo Casanova du 30 avril 1797 (Marr $8-28)$.

49. Je fais référence ici à la lettre envoyée par Casanova de Dux, le 2 juin 1797 dans Casanova 1969, 424-27.

50. Lettre de Cecille von Roggendorff à Giacomo Casanova de Kassovi, le 15 juin 1797 (Marr 8-112). 
uniquement dans son imagination. Mais Cecille est trop accoutumée aux souffrances pour s'abandonner à des idées chimériques. Dès qu'elle a quitté le couvent, défini comme un "asile heureux séjour de l'innossance [innocence]", les vicissitudes de la vie lui ont fait bientôt comprendre qu'elle n'aurait dû chercher le bonheur en dehors d'elle-même. De plus, elle affirme avoir vu clairement les choses depuis le début, mais elle n'avait pas eu le courage de s'expliquer avec lui. Elle ne peut donc compter sur des bienfaits plus réels; probablement emporté par un sentiment de pitié, Casanova lui a manifesté ce qu'elle aurait voulu se cacher à elle-même: "soyez reconnoissante au comte de Waldstein, précisément parcequ'il ne vous a pas répondu, car s'il vous avait répondu, il n'aurait pas pu s'abstenir de vous donner de espérances, car il a le coeur excellent; mais, hélas, il vous aurait trompé!"'51 Le chevalier de Seingalt devrait au contraire la blâmer, exactement au nom de la lex potentior: "plaignés un panchant [penchant] que la nature a formé du quel je ne suis pas la maitresse, qui est plus fort que moi". ${ }^{2}$

Ce penchant est cependant de courte durée puisque Cecille apprend à son égard des propos peu flatteurs:

Que fait la belle Comtesse? Si elle etoit telle qu'on me l'a depeint, je vouderai l'épouser. C'est à dire jolie, car, pour son morale, je la connoit très bien: elle m'a écrit 5 lettres et a cru se mettre en correspondence avec moi, mais Dieu me garde car, quoi qu'elle à de l'esprit, elle est une petite romanesque qui m'ennuit. Pour me défaire de ses importunités, j’ai engagé et ordonné à Casanova, qui conduit toutes mes correspondence, de lui écrire et il me communique toutes ces lettres; pour son frère, le gueux qui vit de mes bienfaits, c'est le plus grand vorien [vaurien] qui puisse. . . .53

Non seulement Ernst a perdu sa réputation, mais Cecille a pu se rendre compte que l'idée donnée par Casanova du caractère du comte de Waldstein n'était pas si mensongère: “j'etoit loin de le croire aussi vil, aussi bas. Cependant il a promis à la même dame de venir surement à Cassovie

51. Lettre de Giacomo Casanova à Cecille von Roggendorff de Dux, le 12 mai 1797 (Marr 40-3).

52. Lettre de Cecille von Roggendorff à Giacomo Casanova de Kassovi, le 15 juin 1797 (Marr 8-112).

53. Lettre de Cecille von Roggendorff à Giacomo Casanova de Sarkösz, le premier août 1797 (Marr 8-29). 
pour me voir, tachés de l'en depersuader, car j'ai si peu d'envie de le voir que de l'épouser" (Marr 8-29).

Cecille reste comme dame d'honneur à la cour du duc Pierre de Curlande moins d'une année, avant de rentrer à Vienne. Le 12 juillet 1802, elle épousera le comte Antal Batthyani-Straettman (1762-1828) et le couple aura quatre enfants: Gusztav (1803), Filippin (1805), Kazmer (1807) e Eleonora (1808). L'époux de la princesse Clary, le comte Charles Clary, postilla, le premier décembre 1814, la mort de Cecille (survenue le 27 novembre) dans son journal avec ces mots:

Madame Fuchs etoit très jolie. Elle avoit sur la tête un magnifique diadème extremement large appartenant a cette pauvre madame Battyany morte il y a 3 jours. Lorsque madame Fuchs l'avoit demandé, sans doute la pauvre femme vivoit encore, mais il y avoit quelque chose de cruel a le voir sur cette tête aujourd'hui. Il me paroit qu'a la place de madame Fuchs j'aurois mieux m'en passer et avec un peu moins de diamants sur le corps.

Università di Pisa

\section{Works Cited}

Anonymous. 1767. Lettres de Ninon de Lenclos au marquis de Sévigné, 2 vols. Amsterdam-Paris: Joly.

Bartolini, Elio. 1994. Casanova. Dalla felicità alla morte 1774-1798. Milano: Mondadori.

Battistini, Andrea, and Ezio Raimondi. 1990. Le figure della retorica. Una storia letteraria italiana. Torino: Einaudi.

Bressy, Joseph. 1789. Recherches sur les vapeurs. Paris: Planche.

Brunot, Ferdinand. 1905. Histoire de la langue française des origines à 1900. Paris: Colin.

Capaci, Bruno, and Elena Grazıoli. 1996. "Il tavolino della dama, lettere e letture di Caterina Dolfin". Studi veneziani 21: 191-228.

- 2019. Giacomo carissimo. . . Lettere delicate e deleterie a Giacomo Casanova, foreword by Piermario Vescovo. Bologna: I Libri di Emil.

Casanova, Giacomo. 1969. Epistolario (1759-1798), edited by Piero Chi ar a. Milano: Longanesi.

—. 2013-2018. Histoire de ma vie, edited by Jean Christophe IGALENs and Érik Leborgne, 3 vols., Paris: Laffont.

Сатасн, Nina. 2001. Histoire de l'orthographe française. Paris: Champion. 
Cengrarotti, Giuseppe. 1990. Gli ultimi anni di Giacomo Casanova in Boemia. Note storiche (1785-1798). Firenze: Atheneum.

Chemello, Adriana, ed. 1998. Alla lettera. Teorie e pratiche epistolari dai Greci al Novecento. Milano: Guerrini.

Damerini, Gino. 1929. La vita avventurosa di Caterina Dolfin Tron. Milano: Mondadori.

Delon, Michel. 2011. Le principe de délicatesse. Libertinage et mélancolie au XVIII siècle. Paris: Albin Michel.

FORNER, Fabio. 2020. Scrivere lettere nel XVIII secolo: precettistica, prassi e letteratura, foreword by Amedeo Quondam. Verona: QuiEdit.

FORNER, Fabio et al., eds. 2017. Le carte false: epistolarità fittizia nel settecento italiano. Roma: Edizioni di Storia e Letteratura.

Grosse, Sybille. 2017. Les manuels épistolographiques français entre tradition et norme. Paris: Honoré Champion.

Korneeva, Tatiana, ed. 2019. Il tappeto rovesciato. La presenza del corpo negli epistolari e nel teatro dal XV al XIX secolo. Venezia: Marsilio.

Ligne, Charles-Joseph De. 2003. Caractères et portraits, edited by Danielle Acke. Paris: Honoré Champion.

Leeflang, Marco. 2000. "Le prince de Ligne, Casanova et l'immortalité de l'âme d'Élise von der Recke". Nouvelles annales Prince de Ligne 14: 133-43.

Mortara Garavelli, Bice. 2018. Manuale di retorica. Milano: Bompiani.

Orsenigo, Vittorio. 1997. A Giacomo Casanova: Lettere d'amore di Manon Balletti ed Elisa von der Recke. Milano: Archinto.

Piazza, Francesca. 2004. Linguaggio persuasione e verità. La retorica del Novecento. Roma: Carocci.

- 2015. La retorica di Aristotele. Introduzione alla lettura. Roma: Carocci.

Perelman, Chaïm, and Lucie Olbrechts-Tyteca. 2008. Traité de l'argumentation. La nouvelle rhétorique. Bruxelles: Édition de l'université de Bruxelles.

Pizzamiglio, Gilberto, ed. 2001. Giacomo Casanova tra Venezia e l'Europa, Proceedings of the conference Giacomo Casanova tra Venezia e l'Europa (Venezia, 16-18 novembre 1998). Firenze: Olschki.

Plebani, Tiziana. 2019. Le scritture delle donne in Europa: pratiche quotidiane e ambizioni letterarie. Secoli XIII-XX. Roma: Carocci.

Pollio, Joseph, and Raoul VÈze. 1926. "La dernière amie de Jacques Casanova: Cécile de Roggendorff (1797-1798)”. Pages Casanoviennes 6.

Ravà, Aldo. 1912. Lettere di donne a Casanova. Milano: Treves.

Roggendorff, Cécile de. 2005. Lettres d'amour à Casanova, edited by Alaine BuISINE. Paris: Zulma.

Rollin, Sophie. 2009. "Les lettres de Vincent Voiture: du discours familier au texte littéraire”. Studi francesi 158: 251-63.

Sta robinski, Jean. 2012. L'encre de la mélancolie. Paris: Seuil.

VAssalli, Sebastiano. 2002. Dux: Casanova in Boemia. Torino: Einaudi. 
Viola, Corrado, ed. 2011. Le carte vive: epistolari e carteggi nel Settecento. Roma: Edizioni di Storia e di Letteratura.

Voiture, Vincent, 1650. Euvres de M. de Voiture, edited by Martin Pinchesne. Paris: Augustin Courbé.

Voltaire. 1830-1834. CEuvres de Voltaire. Correspondance, 19 vols., edited by M. Beuchot. Paris: Firmin Didot frères. 